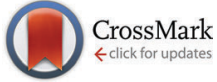

Cite this: Phys. Chem. Chem. Phys., 2015, 17, 21880

Received 8th May 2015

Accepted 28th July 2015

DOI: $10.1039 / c 5 c p 02676 h$

www.rsc.org/pccp

\title{
Atomistic modeling of a KRT35/KRT85 keratin dimer: folding in aqueous solution and unfolding under tensile load
}

\author{
Patrick Duchstein, Tim Clark and Dirk Zahn*
}

\begin{abstract}
We present an atomistic model of a full KRT35/KRT85 dimer, a fundamental building block of human hair. For both monomers initial structures were generated using empirical tools based on homology considerations, followed by the formulation of a naiive dimer model from docking the monomers in vacuum. Relaxation in aqueous solution was then explored from molecular dynamics simulation. Driven by hydrophobic segregation and protein-protein hydrogen bonding relaxation dynamics result in a folded dimer arrangement which shows a striking encounter of cystein groups. Our simulations hence suggests that (i) cystein groups in the coil regions of keratin are well suited to establish disulfide bonds between the two monomers that constitute the dimer, and (ii) the particularly large number of cystein groups in the head and tail regions promotes the connection of dimers to establish meso- to macroscale fibers. Moreover, we show the molecular mechanisms of elastic and plastic deformation under tensile load. Upon elongation beyond the elastic regime, unfolding was identified as the exposure of hydrophobic moieties and the breaking of protein-protein hydrogen bonds. Therein, the step-wise character of the series of unfolding events leads to a broad regime of constant force in response to further elongation.
\end{abstract}

\section{Introduction}

Keratin proteins are key constituents of everyday bio-fibers and tissue such as hair, wool and skin layers. Despite their tremendous importance, which ranges from life science to textile technology, there is still little in-depth knowledge of the molecular structure of keratin fibers. While the primary sequences of the different keratin types are well-established, the assembly into (hetero-)dimers, higher oligomers and microfibrils is only sparsely understood. This particularly applies to the less-ordered linker domains, which account for inter-protein bonds and thus are of key importance in determining mechanical properties.

Yet we are still at the beginning of developing a bottom-up understanding of the molecular structure of keratin dimers and microfibrils. Both hair and skin are tissue constituents of animated organism and subject to a variety of functions which are closely connected not only to structure, but also dynamical processes. The latter involve shielding/penetration of molecules, chemical reactivity and mechanical properties. Apart from sole elasticity which could be achieved by a manifold of soft tissue

Lehrstuhl für Theoretische Chemie/Computer Chemie Centrum, Friedrich-Alexander Universität Erlangen-Nürnberg, Nägelsbachstraße 25, 91052 Erlangen, Germany.

E-mail:dirk.zahn@fau.de types, evolution has lead to a remarkable combination of both elasticity (upon gentle mechanical loading) and resilience against fracture. To identify the fundamental mechanisms of fiber/tissue elasticity, creep, fatigue/self-healing and chemical damage it is of crucial importance to support experimental evidence by molecular modeling and simulation techniques. Indeed, molecular dynamics simulations seem particularly suited to unravel the interplay of hydrogen bond and disulfide bridge dissociation and reformation during mechanical load and self-healing processes that account for the remarkable resilience of hair. In what follows we take a first step in this direction, i.e. create an atomistic model of a KRT35/85 (the most abundant keratin species in human hair) hetero-dimer and demonstrate the analysis of mechanical properties by means of molecular dynamics simulations. Moreover, we aim at elucidating the molecular scale processes of both damage upon loading beyond the elastic limit and explore possible mechanisms of selfhealing by molecular reorganization.

\section{Models and methods}

\section{Experimental benchmarks available}

Keratin proteins are devised in two types which fold as heterodimers that can be connected to form a micro-fibril - the building block to larger fibrils. While the primary sequence 


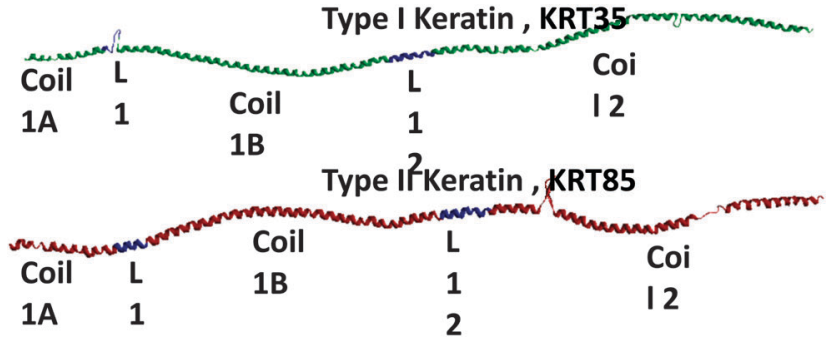

Fig. 1 Two "naïve" keratin monomer coils as prepared from homology modeling. The missing head and tail groups are elusive to homology modeling and were added as random folds and then subjected to relaxation in aqueous solution (see also Fig. 2).

of keratin type proteins is well documented, e.g. in the uniprot database, knowledge of the explicit molecular structure is rather sparse. In lack of information from crystal structure refinement (keratin dimers do not form crystals), so far only approximant models were subjected to molecular modelling. ${ }^{1-4}$ Therein, the well-established alpha-helical parts of keratin dimers were considered (these fragments could be made accessible to crystal structure refinement ten years ago ${ }^{5}$ ), leaving out the less-ordered head, tail and the linker fragments (see also Fig. 1).

Apart from experimental information on the peptide sequences, X-ray diffraction also provided some knowledge of the structure of keratin assemblies. Despite the globular structure of the head and tail regions (and limited structural information on the linkers), the overall assembly of two different keratin molecules into a heterodimer is established for more than 50 years by Pauling et $a l^{6}$ postulating the coiled coil structure. Two alpha helices are coiled into a superstructure, exhibiting a recurring motif of seven amino acids, with hydrophic amino acids in positions 1 and 4. These intermolecularly interacting amino acids provide for the stabilization of the dimer. ${ }^{6}$ Recent X-ray analyses confirmed a monomermonomer distance of $\sim 0.5 \mathrm{~nm}$ within these hetero-dimers. ${ }^{7}$ Moreover, tetramer type bundles have been characterized as comprising of two hetero-dimer strands which are closely connected $(1.3 \mathrm{~nm}$ distance). ${ }^{8}$ From assemblies of tetramer filaments, microfibrils, comprising around 30 filaments are formed, whilst the macroscopic fibril is considered as assemblies of microfibrils [see ref. 8 and references therein]. An important feature of this hierarchical structure is represented by the possibilty to alter clock-wise and anti-clock-wise coiling of keratin monomers into the heterodimer, two heterodimers into a filament, several filaments into a microfibril and different senses of rotation in 'woving' microfibrils into fibers. However, structural information at microfibril length scale $(\sim 70 \mathrm{~nm}$ diameter) is still limited. Indeed, most recent X-ray analyses indicate a parallel (non-woven) arrangement of dimers into tetramers, whilst several tetrameters are assumed to be coiled into a microfibril. ${ }^{8}$

In lack of detailed structure data from keratin crystals the molecular models discussed in the present work were created along a two-step strategy. We (i) employ bioinformatics to create a starting model and then use (ii) all-atom detailed molecular dynamics simulations to explore system relaxation to realistic configurations in aqueous solution.

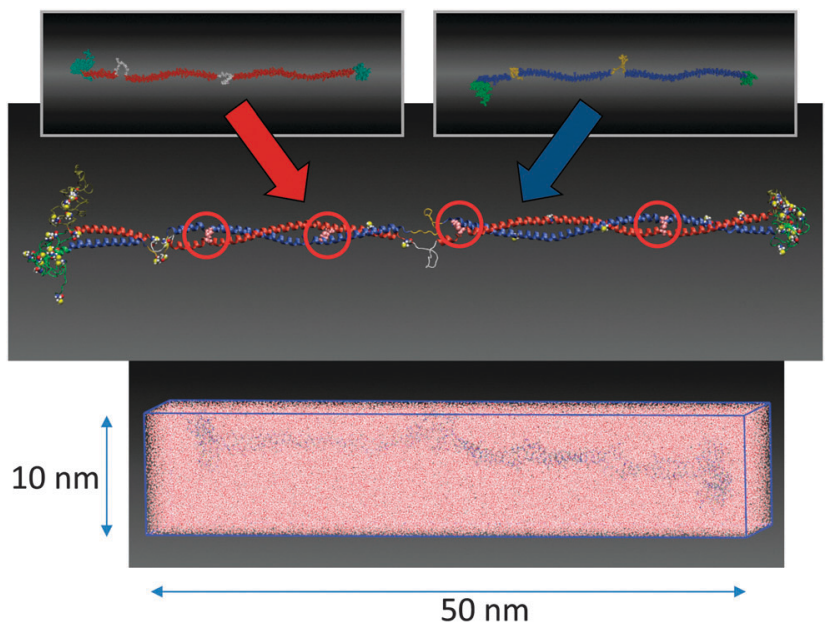

Fig. 2 Keratin monomers as pre-modeled in vacuum (top), assembled as a dimer (center) and folded in aqueous solution (bottom). The KRT35/85 dimer consists of helical domains that are believed to determine the stiffness of the biomolecules, head/tail domains that account for dimerdimer bonds and linker domains, whose function is poorly understood. While the protein backbones are shown as ribbons, the atoms of the cystein groups are illustrated by spheres.

\section{Preparation of starting models}

Keratin bio-fibers consist of a hierarchical assembly of a variety of keratin proteins. The fundamental building-block is constituted of protein dimers, in which different keratin types are involved depending on the designated function of the biomaterial (e.g. skin or hair). In wool and hair, a prototypical hetero-dimer can be modeled on the basis of the monomers KRT35 and KRT85. In lack of detailed structural information, here we present a model of the Keratin KRT35-KRT85 dimer, starting from the UniProt sequences Q92764 and P78386 (Fig. 1). In the initial configuration the coiled coil regions $1 \mathrm{~A}, 1 \mathrm{~B}$, and 2 were set up in a coiled coil fashion with a pitch of 0.51 and 3.5 amino acids per turn, respectively. For the flexible linker regions a random coil was chosen as starting configuration. No homologs to the head- and tail groups could be identified. We therefore created the starting models of the head- and tail groups of the keratin monomers via threading. This way of starting configuration preparation uses 'local' homology based on segments of the protein. While we used I-TASSER ${ }^{9}$ to prepare optimal starting models, we point out that reliable conformations of large proteins require in-depth relaxation from molecular simulation as described in the following section.

\section{Model relaxation/molecular dynamics simulations}

All-atom detailed molecular dynamics simulations are employed to allow the system to relax from the starting models based on homology considerations. Protein dimer self-organization results from the interplay of favorable hydrogen bonding patterns between the proteins and the segregation of hydrophobic moieties. Thus, the initial dimer model was immersed into aqueous solution and subjected to relaxation from molecular dynamics simulations. At this stage, we rely on full atomistic 
models which are intrinsically complex, as large biomolecules (416 to 507 amino acids per monomer) are investigated in aqueous solution $(\sim 150000$ water molecules needed for solvating the dimer, see also Fig. 2).

The molecular dynamics simulations were performed in the constant-pressure, constant-temperature ensemble, imposing ambient conditions. The molecular interactions were described by the AMBER ff12SB force-field. ${ }^{10}$ The accuracy of this state-ofthe-art force-field was very recently confirmed for a large series of benchmarks focused on both, protein backbones and side chains. ${ }^{11}$ A time-step of 2 fs was applied. Because of the large dimensions of the simulation cell $\left(\sim 10 \times 10 \times 50 \mathrm{~nm}^{3}\right)$ the calculation of long range Coulomb interactions does not require Ewald summation (note that the $k$-space summation would refer to interactions beyond distances of $10 \mathrm{~nm}$ ) and a $1 \mathrm{~nm}$ shifted force cut-off potential was employed. After immersing the initial keratin dimer model into aqueous solution, we first kept the biomolecule fixed while the water molecules are allowed to adopt within a $10 \mathrm{~ns}$ simulation run. Overall model relaxation was then investigated from $1.77 \mu$ s unconstrained molecular dynamics simulation.

To monitor the relaxation process we analyzed the number of hydrophobic residues exposed to the solvent and the overall head-to-tail distance of the dimer. While the former was found to relax within a few tens of picoseconds, the latter reflects the more critical observable. Indeed, the head-to-tail distance reflects the sum of many folding events and changed from 40 to about $30 \mathrm{~nm}$ within $100 \mathrm{~ns}$. After this relaxation, the unconstraint dimer still tends to bend and unbend as a fluctuating process that full account would require longer simulations. However, as our focus is dedicated to a dimer model as a building block within a biofiber, we decided to prepare an elongated, yet not stretched, dimer model as discussed below.

Starting from the relaxed model obtained from $1.77 \mu$ s of unconstrained molecular dynamics simulations we implemented a bias with respect to dimer elongation - at this stage without the application of tensile load. For this purpose, in principle unconstrained molecular dynamics simulations were devised in series of 100 ps runs. For each time interval two independent runs were performed using different velocity distributions (based on a simulation temperature of $300 \mathrm{~K}$ ). For both runs, we compared the final head-to-tail distance of the dimer models. The run of larger head-to-tail distance was then used as the starting point for another 100 ps run. Along such iterations, we implemented a sampling bias that fully compensates the entropic favoring of coiled arrangements, but does not (yet) apply tensile load. Using this strategy, convergence of the head-to-tail distance to $39 \mathrm{~nm}$ was found from a total of $320 \mathrm{~ns}$ molecular dynamics runs.

\section{Results}

\section{Solvated dimer}

Keratin dimer relaxation in aqueous solution was found to involve local rearrangements whilst the overall structure, namely the coiled coil alignment of the proteins persists. Driven by hydrophobic segregation and protein-protein hydrogen bonding relaxation dynamics result in a folded dimer arrangement which shows a striking encounter of cystein groups (Fig. 2, center). Our simulations hence suggests that (i) cystein groups in the coil regions of keratin are well suited to establish disulfide bonds between the two monomers that constitute the dimer, and (ii) the particularly large number of cystein groups in the head and tail regions promotes the connection of dimers to establish meso- to macroscale fibers.

It is noteworthy that there are striking differences concerning the peptide sequences of keratin types found in skin and hair. While keratin in hair (mainly KRT 35/85 dimers) are rich in cystein groups, cystein is practically absent in epidermal keratin (KRT 10/1 and KRT 14/5). As a consequence, keratinkeratin disulfide bridges are expected to account for differences both in terms of structure and mechanical properties.

To provide a realistic account of the mechanical properties of the folded dimer, cystein groups along the coil regions that are at sufficiently close distance were assumed to form disulfide bonds. Based on a distance delimiter of $1.5 \mathrm{~nm}$ we identified 4 pairs of cystein residues (K35C160-K85C185, K35C213-K85C234, K35C271-K85C290, K35C357-K85C383, highlighted by red circles in Fig. 2, center) that are expected to establish disulfide bridges between the two keratin monomers. These bonds were thus established in our molecular model as used for all further investigations.

To allow comparison to the available experimental data, we calculated the theoretical X-ray diffraction function of the keratin dimers. X-ray diffraction data is only available for hair samples, not individual dimers. Nevertheless, these experiments provide a series of characteristic distances, including the monomermonomer distance at finest resolution. Our simulation models show good agreement with the experimentally assessed value of $0.5 \mathrm{~nm}$ (Fig. 3).

As part of the analysis of keratin dimer relaxation in aqueous solution we furthermore explored local affinity to binding water molecules. All protein-water hydrogen bonds were subjected to life-time statistics which ranges from 1 to $\sim 25$ picoseconds. On this basis, particularly hydrophilic regions were identified

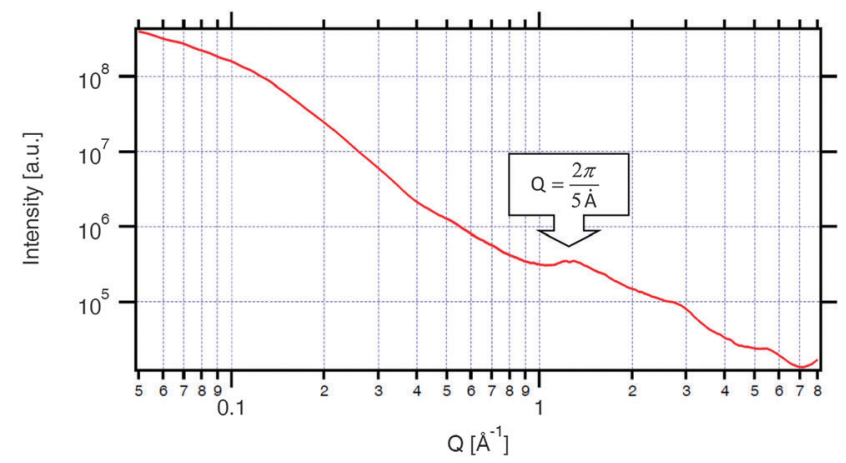

Fig. 3 Theoretical X-ray diffraction function as calculated for the relaxed keratin dimer shown in Fig. 4. Comparison to experimental data clearly confirms the $0.5 \mathrm{~nm}$ signal which is interpreted as the average monomermonomer distance. ${ }^{8}$ 
within the head, tail and linker regions of the keratin dimers as highlighted in Fig. 4.

\section{Tensile loading}

In molecular dynamics simulations mechanical load may be applied by either considering external forces or by imposing distance constraints. For complex model systems direct application of external forces may lead to strong hysteresis effects. We therefore imposed mechanical load using (head-to-tail) distance constraints at a variety of strain rates. Considering the 1-100 microsecond-scales accessable to molecular-dynamics simulations of million-particle systems, the modeled strain rates are typically larger than those used in the experiment. A series of rates was therefore tested to quantify the dependence of our results on strain-rate. On this basis, a strain rate of $5 \mathrm{~m} \mathrm{~s}^{-1}$ was found suitable for deriving a consistent stress-strain diagram as discussed in the following.

The dimers constitute the smallest building blocks of hair fibers. Nevertheless, the stress-strain diagram as illustrated in Fig. 5 already shows a typical signature of tissue materials, i.e. a broad elastic regime and an even more pronounced viscoelastic/creep regime. Starting (i) from the dimer model as obtained from relaxation with bias to spontaneous elongation to compensate for coiling driven by entropy, the elastic regime was found up to elongation by $7.5 \mathrm{~nm}$ (ii). This process is characterized by the stretching of the linker domains, whilst the coil regions of the dimer remain practically unchanged (Fig. 6).

After elongation beyond the yield strain we observe a roughly constant force response which can be rationalized by the

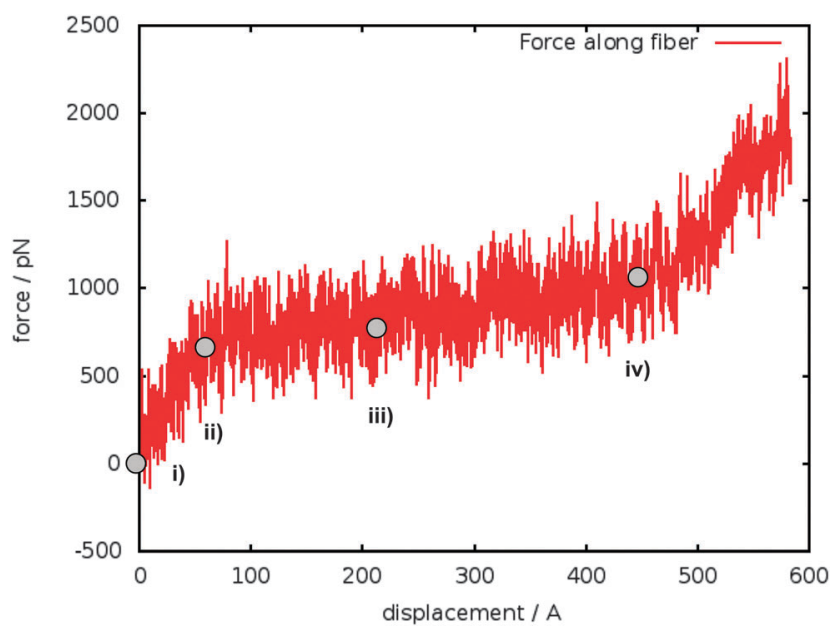

Fig. 5 KRT35/85 dimer under tensile load. Elongation beyond the yield strain $\left(7.5 \mathrm{~nm}, \varepsilon_{y}=19 \%\right)$, leads to a regime of roughly constant force with is related to the gradual dissociation of protein-protein hydrogen bonds.

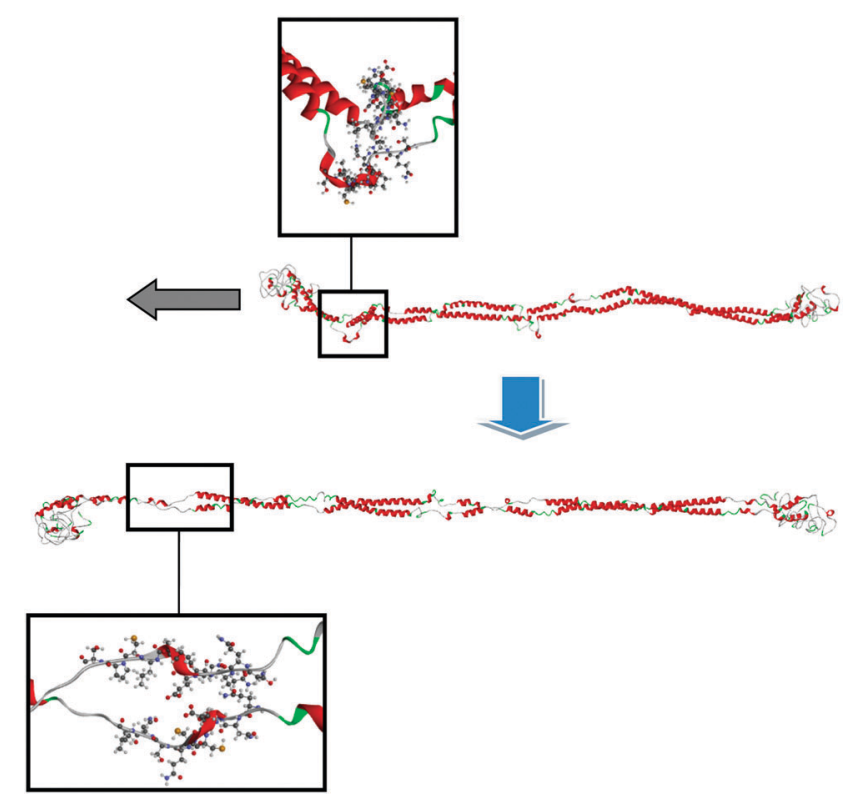

Fig. 6 Pristine keratin dimer (top) and dimer structure under tensile loading up to the yield strain (bottom). Drastic structural rearrangements are observed for the linker domains only, whilst the coil regions exhibit only marginal deformation.

gradual dissociation of hydrogen bonds within the protein. This process may be nicely illustrated by assessing the number of hydrogen bonds in different manners, i.e. on the basis of donor-acceptor distances, only, and on the basis of both distance and the $\mathrm{R}-\mathrm{O} \cdots \mathrm{H}$ angles. As illustrated in Fig. 7a, we find hydrogen bond dissociation only after elongation by $\sim 20 \mathrm{~nm}$ (iii), whilst a decline in hydrogen bonding strength by bending the $\mathrm{R}-\mathrm{O} \cdots \mathrm{H}$ angles is observed immediately after loading beyond the yield strain (ii). Upon tensile loading of the keratin dimer beyond $45 \mathrm{~nm}$ (iv) all hydrogen bonds are broken, and we observe a steep increase in the stress-strain diagram which may be related to the unwinding of the alphahelices of the coil domains.

It is noteworthy that dimer dissociation, i.e. full dissociation of all hydrogen bonds connecting the monomers, requires rather drastic elongation. Up to about $45 \mathrm{~nm}$, tensile loading does not destroy the overall structural integrity of the dimer. We explored the relaxation of the dimer model from $100 \mathrm{~ns}$ unconstrained molecular dynamics simulations at ambient conditions starting from configurations (iii) and (iv) referring to 20 and $45 \mathrm{~nm}$ elongation, respectively. Within these relaxation runs the keratin dimer only partially recovered from elongation. Indeed, the head-to-tail distances rapidly decreased by about $10 \mathrm{~nm}$, but then reached a plateau. This is in line with the observation that only some of the hydrogen bonds that were dissociated by tensile 

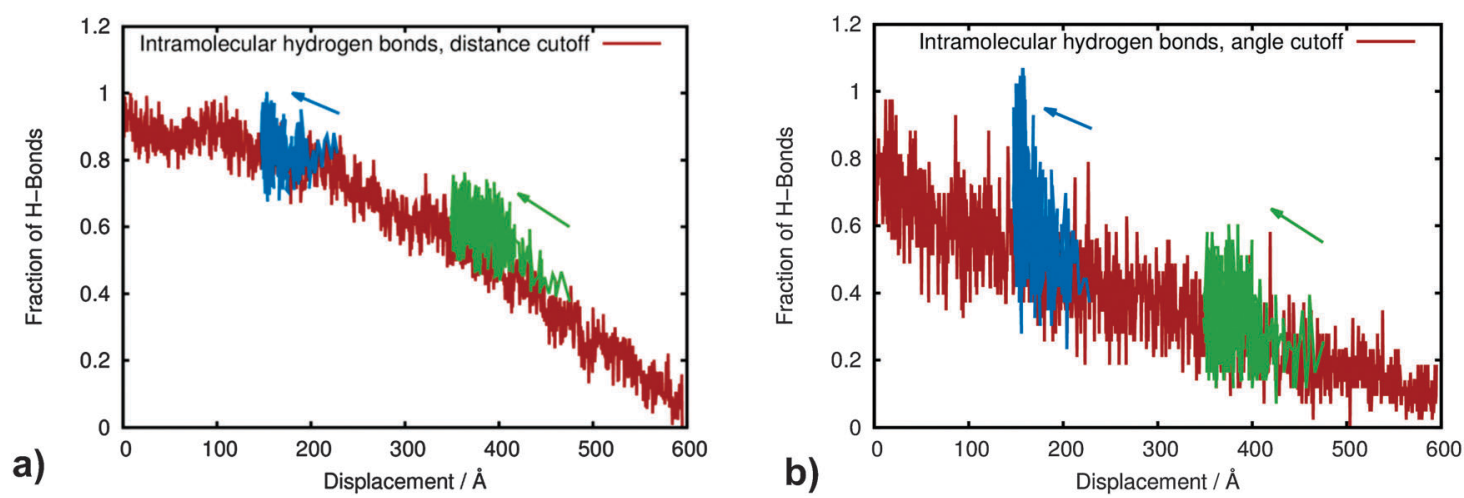

Fig. 7 Fraction of keratin-keratin hydrogen bonds as a function of tensile loading as assessed on the basis of (a) donor-acceptor distance of less than $0.3 \mathrm{~nm}$, and (b) additionally using a delimiter of angular deformation by less than $10^{\circ}$. The blue and green lines denote the fraction of recurring hydrogen bonds during relaxation of the dimer model starting from configuration (iii) and (iv), respectively (see Fig. 5).

loading beyond yield strength actually recovered within the $100 \mathrm{~ns}$ runs. The non-elastic response of the keratin dimer may thus be related to the gradual re-establishing of hydrogen bonds.

\section{Conclusion}

An atomistic model of a full KRT35/KRT85 dimer was prepared from homology modelling followed by relaxation in aqueous solution explored from $1.77 \mu$ s molecular dynamics runs. On this basis we find a series of nearby cystein groups in the coil regions of keratin which are expected to establish disulfide bridges between the two monomers and thus stabilize the hetero-dimer. The resulting structural model of the dimer constitutes a starting point for constructing hierarchical models of keratin fibers and microfibrils. While such model sophistication is subject to yet ongoing work, by the example of the KRT35/KRT85 dimer we demonstrated the in-depth analysis of the molecular mechanisms that account for elastic and plastic deformation under tensile load. Upon elongation beyond the elastic regime, protein-protein hydrogen bonds are deformed and eventually dissociated in a step-wise manner.

This molecular scale process accounts for the transformation of mechanical stress into heat as characteristic to plastic deformation. Strikingly, the gradual sacrifice of hydrogen bonding allows the keratin dimer to maintain its overall structural integrity up to elongation by almost $100 \%$. Thus, when releasing mechanical load, the dimer may re-fold and eventually recover to the pristine structure via molecular self-organization. In analogy to similar observations made for collagen and silk fibers ${ }^{12-17}$ we suggest the gradual sacrifice of hydrogen bonding in keratin as a mechanism of self-healing accounting for the remarkable resilience of hair fibers.

\section{Acknowledgements}

We are acknowledging the financial support by the Procter \& Gamble Service GmbH (Sulzbacherstrasse 40; 65824 Schwalbach am Taunus, Germany) in the framework of P\&G's use of modeling in fundamental hair science and scientific contributions of Andreas Schiener from the chair of crystal physics in Erlangen and the Procter \& Gamble scientists Dr Simon Godfrey, Dr Mathias Herrlein, Dr Graham McKelvey and Dr Gabriela Schaefer.

\section{References}

1 T. A. Smith and D. A. D. Parry, J. Struct. Biol., 2008, 162, 139-151.

2 M. Er Rafik, J. Doucet and F. Briki, Biophys. J., 2004, 84, 3893-3904.

3 N. G. Azoia, et al., Proteins, 2012, 80, 1409-1417.

4 J. K. Marzinek, et al., AIChE J., 2013, 59, 4816-4823.

5 S. V. H. Strelkov, et al., EMBO J., 2002, 21, 1255-1266.

6 L. Pauling, R. B. Corey and H. R. Branson, Proc. Natl. Acad. Sci. U. S. A., 1951, 37, 205-211.

7 B. Busson, F. Briki and J. Doucet, J. Struct. Biol., 1999, 125, 1-10.

8 M. Er Rafik, J. Doucet and F. Briki, Biophys. J., 2004, 84, 3893-3904.

9 J. Yang, R. Yan, A. Roy, D. Xu, J. Poisson and Y. Zhang, Nat. Methods, 2015, 12, 7-8.

10 D. A. Case, T. A. Darden, T. E. Cheatham, III, C. L. Simmerling, J. Wang, R. E. Duke, R. Luo, R. C. Walker, W. Zhang, K. M. Merz, B. Roberts, S. Hayik, A. Roitberg, G. Seabra, J. Swails, A. W. Götz, I. Kolossváry, K. F. Wong, F. Paesani, J. Vanicek, R. M. Wolf, J. Liu, X. Wu, S. R. Brozell, T. Steinbrecher, H. Gohlke, Q. Cai, X. Ye, J. Wang, M.-J. Hsieh, G. Cui, D. R. Roe, D. H. Mathews, M. G. Seetin, R. Salomon-Ferrer, C. Sagui, V. Babin, T. Luchko, S. Gusarov, A. Kovalenko and P. A. Kollman, AMBER 12, University of California, San Francisco, 2012.

11 J. A. Maier, C. Martinez, K. Kasavajhala, L. Wickstrom, K. Hauser and C. Simmerling, J. Chem. Theory Comput., DOI: 10.1021/acs.jctc.5b00255.

12 Z. Qin, L. Kreplak and M. J. Buehler, PLoS One, 2009, 4, e7294.

13 S. Keten and M. J. Buehler, Nano Lett., 2008, 8(2), 743-748.

14 A. Nova, et al., Nano Lett., 2010, 10, 2626-2634.

15 T. Giesa, et al., Nano Lett., 2011, 11, 5038-5046.

16 A. Gautieri, S. Vesentini, A. Redaelli and M. J. Buehler, Nano Lett., 2011, 11, 757-766.

17 A. C. Lorenzo and E. R. Caffarena, J. Biomech., 2005, 38, 1527-1533. 